Abstract

\title{
A new method to concentrate equine sperm ${ }^{\text {is }}$
}

\author{
M.A. Alvarenga*, C.M. Melo, L.C.O. Magalhães, F.O. Papa \\ Department of Animal Reproduction and Veterinary Radiology, São Paulo State University, São Paulo, Brazil
}

\section{Introduction}

An ability to increase the concentration of sperm is important in preparing semen for artificial insemination, freezing and cooling, and the removal of seminal plasma and cryoprotective agents (Correa and Zavos, 1996). At present this concentration is usually done by centrifugation. However centrifugation also subjects the sperm to a certain amount of stress, more in some stallions than others (Sieme et al, 2003).

The aim of the present study was to compare two filters for their sperm retention efficiency and their effects on the viability of the sperm after concentration.

\section{Materials and methods}

\subsection{Experiment 1}

Filters were developed by CEAFEPE Tecnologia Veterinária Ind. (Patent US No. 12/529,144). They are $12 \mathrm{~cm}$ in diameter, made up of a hydrophilic synthetic membrane that does not allow the sperm to pass through.

In this experiment eight ejaculates from eight stallions of different breeds were used. The ejaculates were diluted 2:1 (extender:semen) with a milk-base extender (BotuSemen Biotech Botucatu-Brazil), split into three aliquots, and submitted to the following procedures before cooling: (a) filtration with filter A; (b) filtration with filter B, and (c) no filtration, control. Filter A had more pores per

\footnotetext{
is This paper is part of the supplement entitled "Proceedings of the Tenth International Symposium on Equine Reproduction", Guest Edited by Margaret J. Evans.

* Corresponding author at: UNESP, FMVZ, Departamento de Reprodução Animal e Radiologia Veterinaria, Distrito de Rubiao Jr, s/n, Botucatu, SP, CEP 18618-000, Brazil. Tel.: +55 1438824427; fax: +55 1438116326.

E-mail address: malvarenga@fmvz.unesp.br (M.A. Alvarenga).
}

unit of surface area, and consequently filtered more rapidly, than filter B. In the filtered aliquots, sperm concentrations were determined after re-dilution with the same amount of extender that had been present before filtering. The samples were then stored at $15^{\circ} \mathrm{C}$ in a passive cooling device, Botu-Box, for $24 \mathrm{~h}$. After cooling, the semen was incubated in a dryblock at $37^{\circ} \mathrm{C}$ for $10 \mathrm{~min}$ before evaluation. Motility parameters were analyzed by CASA (HTM-IVOS 12) and plasma membrane integrity was evaluated using fluorescent probes (CFDA and PI). Data were analyzed by ANOVA (GraphPad Instat, Version 3.00, 1997) followed by Tukey's test.

\subsection{Experiment 2}

Again, eight ejaculates from eight stallions of different breeds were collected and diluted 2:1 (extender:semen) with Botu-Semen (Biotech Botucatu/ME LTDA, Brazil). However, samples of these ejaculates were stored at $15^{\circ} \mathrm{C}$ in a Botu-Box, for $24 \mathrm{~h}$ before further treatments. After cooling, samples were split into four aliquots and subjected to: (a) sperm retention on filter A; (b) sperm retention on filter B; (c) control; and (d) centrifuged. Evaluation procedures were as in experiment 1 .

\section{Results}

The percentage of sperm cells recovered was $95 \%$ and $92 \%$ for filters A and B, respectively. Motility parameters and membrane integrity from both experiments are shown in Table 1.

\section{Discussion}

Either filter allowed the retention of almost all sperm from the original sample and caused no damage to sperm motility and viability. Thus, the technique is potentially useful, especially with semen of poor quality, for: 
Table 1

Mean \pm SD values for total motility (TM), progressive motility (PM), average path velocity (VAP), straight-line velocity (VSL), curvilinear velocity (VCL) and plasma membrane integrity (PMI) of sperm in equine semen subjected to filtration using different filters (A) and (B), no filtration (control, C), or centrifugation (D) either before (experiment 1) or after (experiment 2) cooling for $24 \mathrm{~h}$ at $15^{\circ} \mathrm{C}$.

\begin{tabular}{|c|c|c|c|c|c|c|}
\hline Group & $\mathrm{TM}$ & PM & VAP & VSL & VCL & PMI \\
\hline \multicolumn{7}{|c|}{ Before cooling, experiment 1} \\
\hline A & $69.9 \pm 11.3$ & $35.0 \pm 8.1$ & $119.5 \pm 18.9^{\mathrm{ab}}$ & $91.5 \pm 13.1$ & $218.0 \pm 25.7$ & $68.5 \pm 6.5$ \\
\hline B & $63.1 \pm 14.0$ & $32.2 \pm 10.7$ & $92.5 \pm 15.8^{\mathrm{a}}$ & $78.6 \pm 12.1$ & $183.5 \pm 36.1$ & $71.5 \pm 16.4$ \\
\hline C & $76.6 \pm 11.1$ & $38.0 \pm 5.4$ & $133.5 \pm 16.7^{b}$ & $91.3 \pm 25.3$ & $222.5 \pm 0.6$ & $68.0 \pm 5.8$ \\
\hline \multicolumn{7}{|c|}{ After cooling, experiment 2} \\
\hline A & $71.0 \pm 14.3$ & $19.5 \pm 9.3$ & $126.0 \pm 28.8$ & $85.5 \pm 6.8$ & $241.0 \pm 18.8$ & $61.0 \pm 8.2$ \\
\hline B & $63.0 \pm 13.6$ & $21.0 \pm 9.5$ & $126.5 \pm 28.7$ & $94.0 \pm 6.6$ & $237.0 \pm 14.1$ & $63.5 \pm 5.0$ \\
\hline C & $79.0 \pm 16.5$ & $25.5 \pm 9.0$ & $126.0 \pm 29.0$ & $95.0 \pm 11.7$ & $235.0 \pm 15.9$ & $61.5 \pm 11.1$ \\
\hline $\mathrm{D}$ & $71.5 \pm 17.9$ & $24.5 \pm 9.1$ & $118.5 \pm 22.0$ & $86.5 \pm 5.0$ & $222.0 \pm 14.7$ & $49.0 \pm 15.1$ \\
\hline
\end{tabular}

Different letters represent different filters, filtration, centrifugation, etc. described in table title.

separating spermatozoa from seminal plasma and/or extender, concentrating sperm for freezing and cooling, and concentrating sperm for low dose AI.

Both filters were efficient in terms of maintaining sperm motility and viability in cooled semen, since there was no difference between the centrifuged and control groups. Sperm from the control group were of excellent quality, probably because the stallions that were used had good semen quality and it maybe that the use of stallions with poor quality semen would show greater benefits of using the filters. In experiment 2 , although not statistically significant, the index of membrane integrity was numerically lower in the centrifuged group than in the filtered groups.
We conclude that the filters can be used to concentrate sperm without reducing motility and viability. Further studies are warranted to determine if this system is a good alternative for obtaining sperm cells from stallions in which the sperm cannot withstand centrifugation.

\section{Conflicts of interest}

None.

\section{Reference}

Correa, J.R., Zavos, P.M., 1996. Preparation and recovery of frozen-thawed bovine spermatozoa via various sperm selection techniques employed in assisted reproductive technologies. Theriogenology 46, 1225-1232. 